\title{
GT2017-63020
}

\section{Impact of Wake Dispersion on Axial Compressor Peformance}

Chunill Hah

NASA Glenn Research Center,

MS 5-10, Cleveland, Ohio 


\section{Background}

- Needs to advance current understanding of flow physics in modern highly-loaded compact compressor stages.

- Needs to develop prediction tools based on higher-fidelity CFD tools (DES, LES, DNS, etc.). 


\section{Changes in multi-stage compressor design}

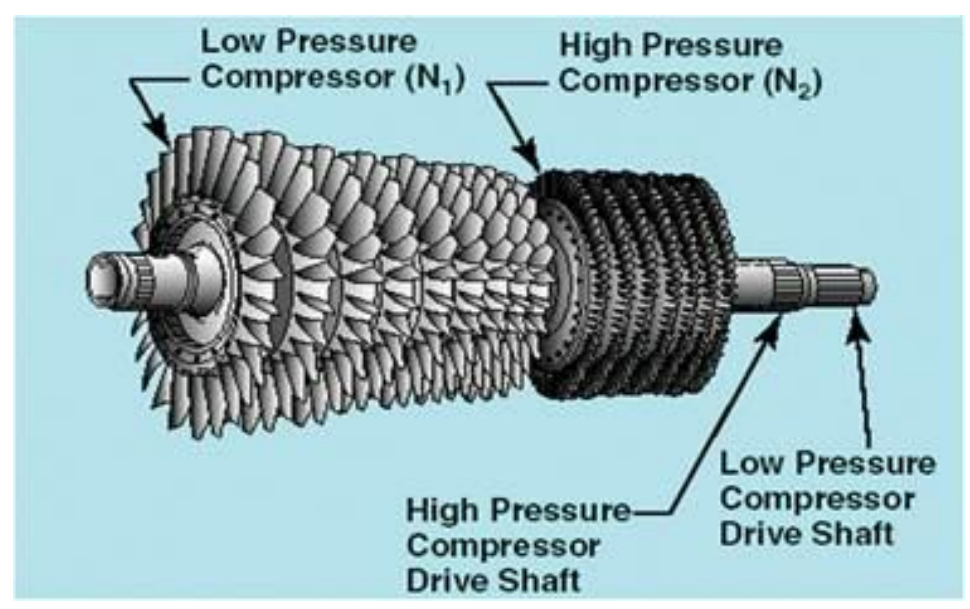

Less spacing between blade rows.

Higher loading per blade rows.

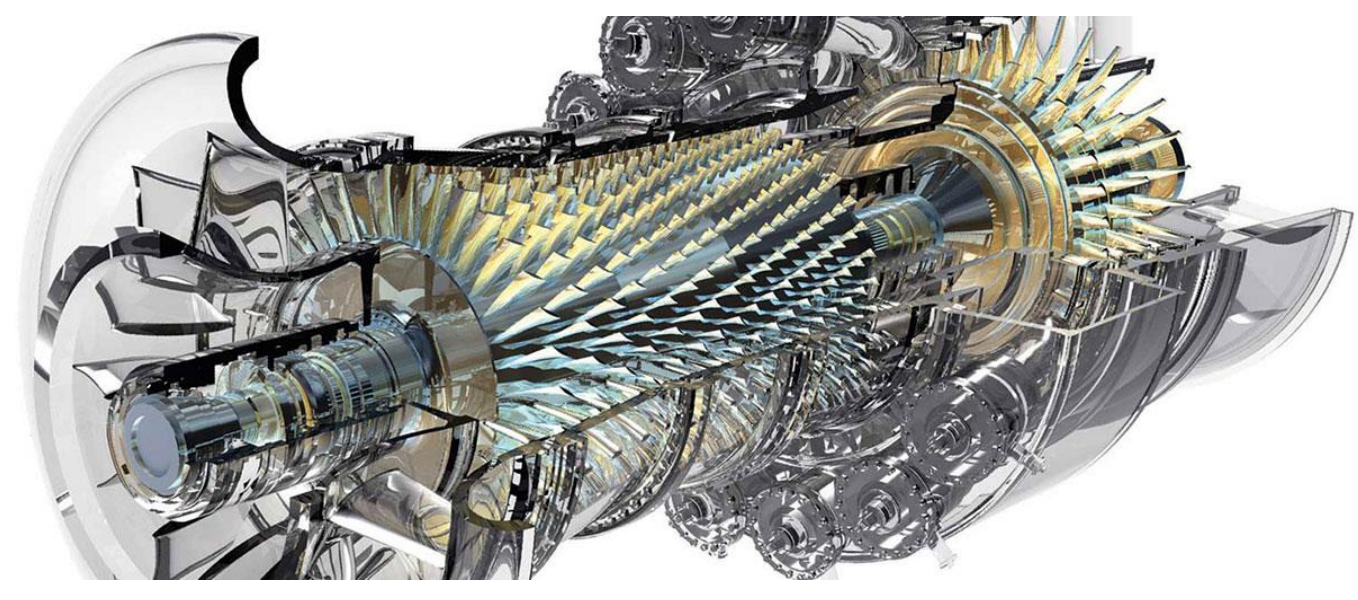



closely-coupled compressor blade rows

1. Upstream influence of pressure field of the downstream blade row.

2. Effects of wake dispersion on the downstream blade row.
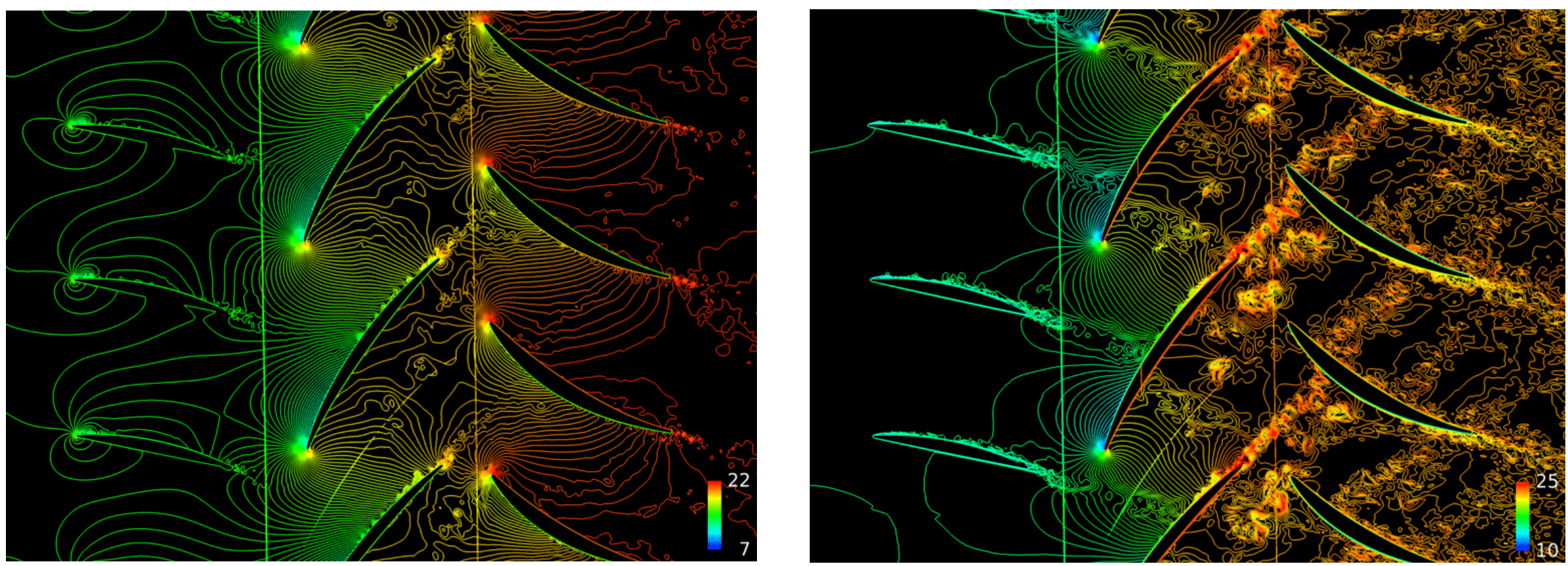

Ps

$\mathrm{Pt}$ 


\section{Earlier investigations of the wake dispersion on compressor performance}

L. H. Smith (1966) : 1.2 points efficiency gain when axial spacing is reduced from 37 to 7 percent of chord.

0.68 points due to upstream pressure effects. 0.52 points due to wake recovery. 


\section{Objectives}

- Investigate effects of wake dispersion on the compressor performance.

- Conducted in one and a half stage axial compressor with two spacing between rotor and stator (112\% and $29 \%$ of rotor axial chord at mid-span).

- LES was applied for the flow simulation. 


\section{Axial compressor stage at JHU test facility}
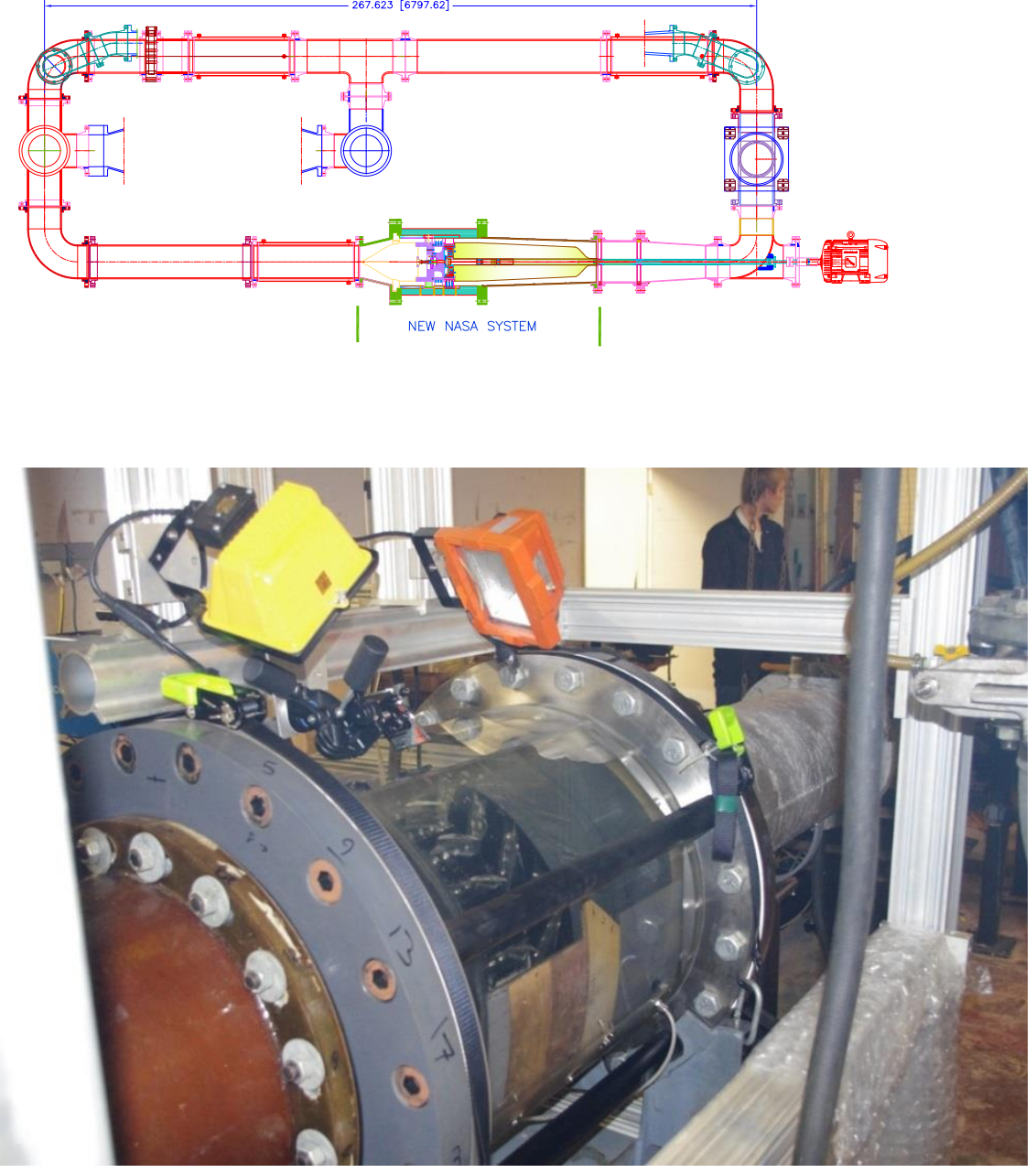

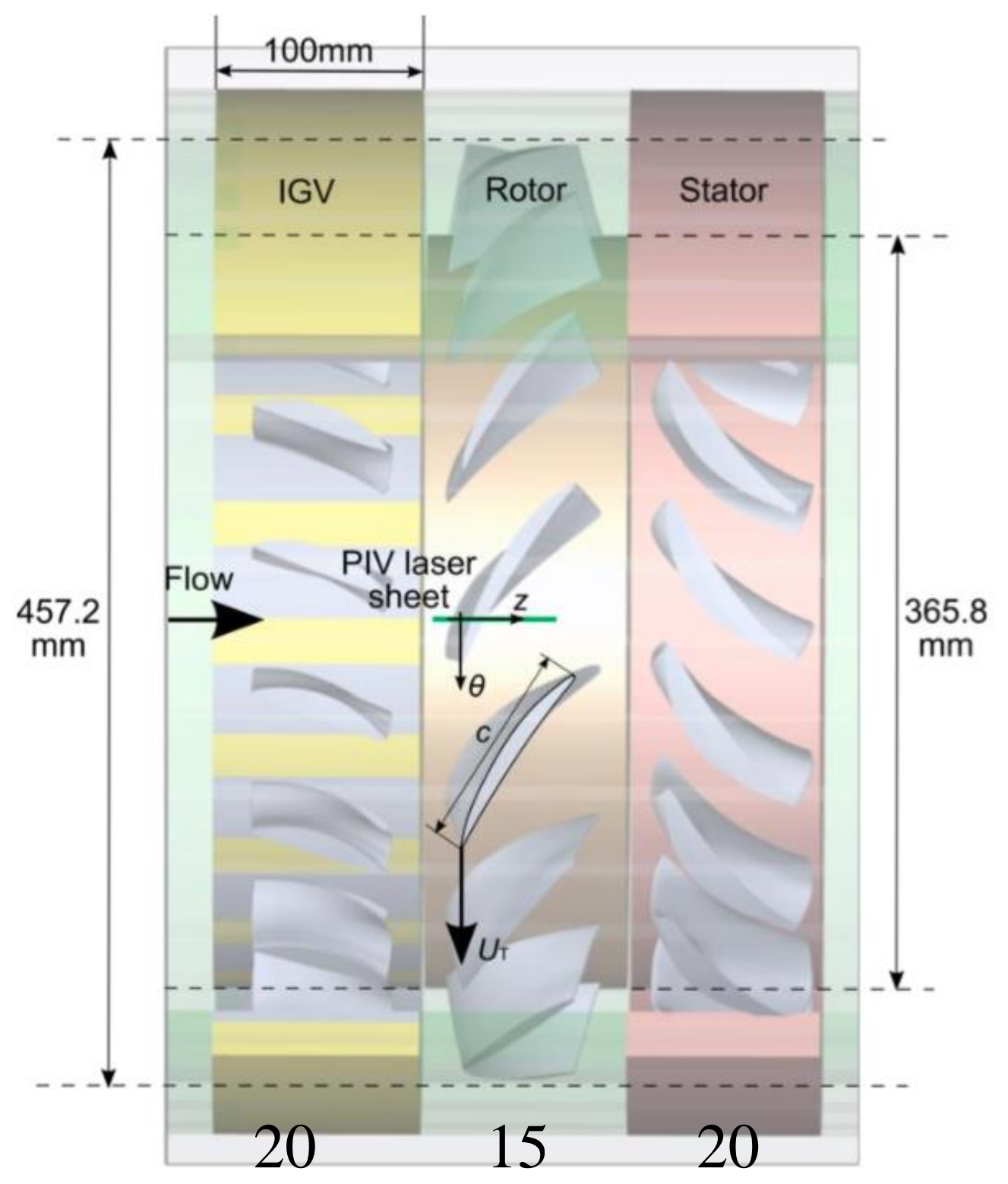




\section{Pressure rise characteristics of the original compressor stage}

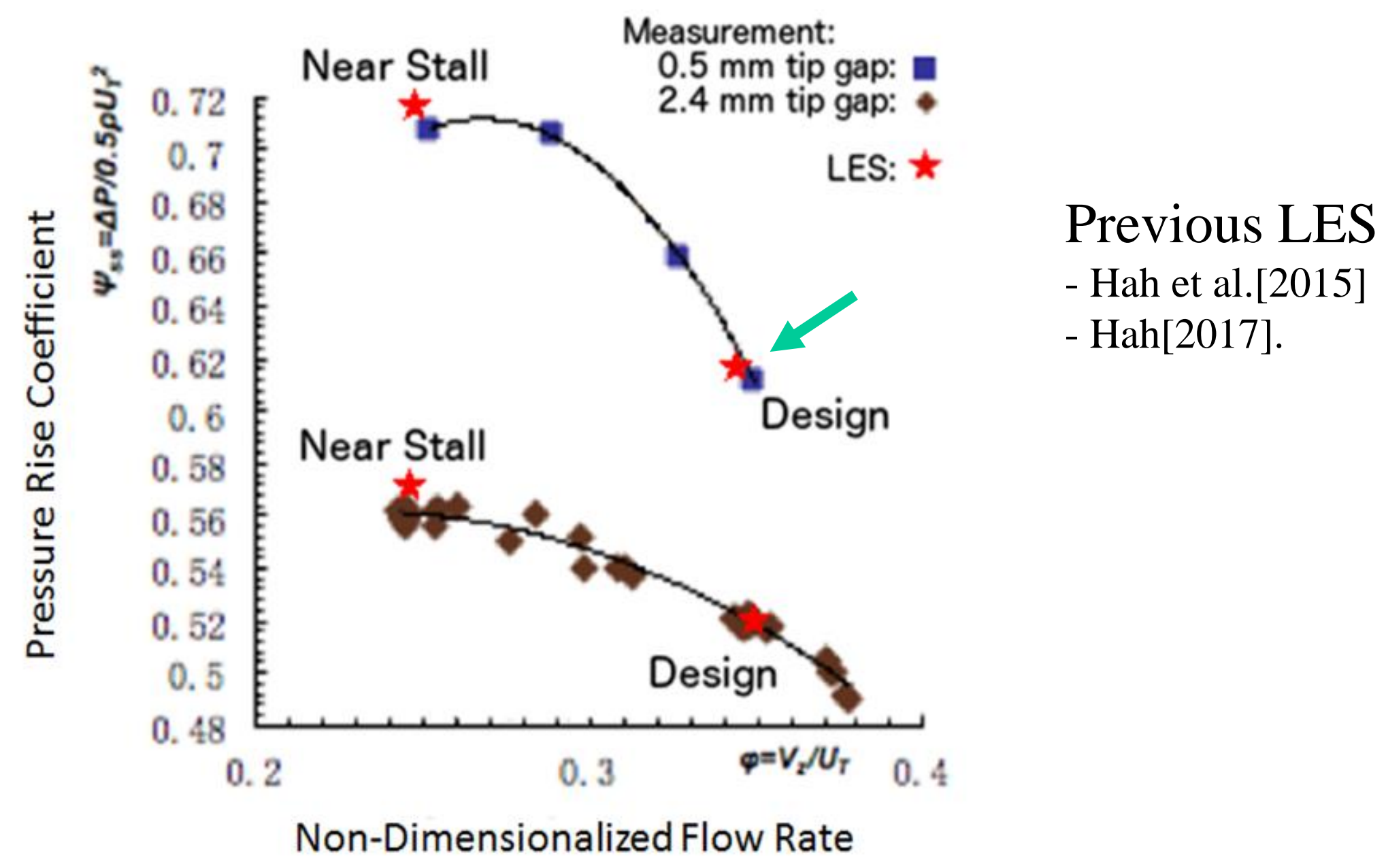




\section{Applied LES procedure}

- $3^{\text {rd }}$-order scheme for convection terms.

- $2^{\text {nd }}$-order central differencing for diffusion terms.

- Sub-iteration at each time step.

- Dynamic model for sub grid stress tensor.

- Multi-block I-grid, 1.2 billion nodes for 4-3-4 simulations with 60 radial nodes inside tip gap. 


\section{Test section and blade geometry}

\begin{tabular}{|c|c|}
\hline Casing Diameter $(D)[\mathrm{mm}]$ & 457.20 \\
\hline Hub Diameter $(d)[\mathrm{mm}]$ & 365.76 \\
\hline Rotor Diameter $\left(D_{\mathbf{R}}\right)[\mathrm{mm}]$ & 455.92 \\
\hline Rotor Blade Chord $(c)[\mathrm{mm}]$ & 102.60 \\
\hline Rotor Blade Span $(\gamma){ }^{[0]}$ & $45.08,43.92$ \\
\hline Rotor Blade Stagger Angle $\left(c_{\mathrm{A}}\right)[\mathrm{mm}]$ & 58.6 \\
\hline Rotor Blade Axial Chord [mm] & 53.46 \\
\hline Nominal Tip Clearance [mm] & $\begin{array}{c}0.64(0.62 \% \text { of } c) \\
1.8(1.75 \%)\end{array}$ \\
\hline Measured Tip Clearance $(h)[\mathrm{mm}]$ & $\begin{array}{l}0.5(0.49 \%) \\
2.4(2.3 \%)\end{array}$ \\
\hline Shaft Speed $(\Omega)\left[\operatorname{rad~s}^{-1}\right]\{R P M\}$ & $50.27\{480\}$ \\
\hline Rotor Blade Tip Speed $\left(U_{T}\right)\left[\mathrm{ms}^{-1}\right]$ & 11.47 \\
\hline Reynolds Number $\left(U_{\mathrm{T}} c / v\right)$ & $1.07 \times 10^{6}$ \\
\hline
\end{tabular}




\section{Cross section of two configurations \\ (tip clearance of $0.5 \mathrm{~mm}, 0.8 \%$ rotor span)}

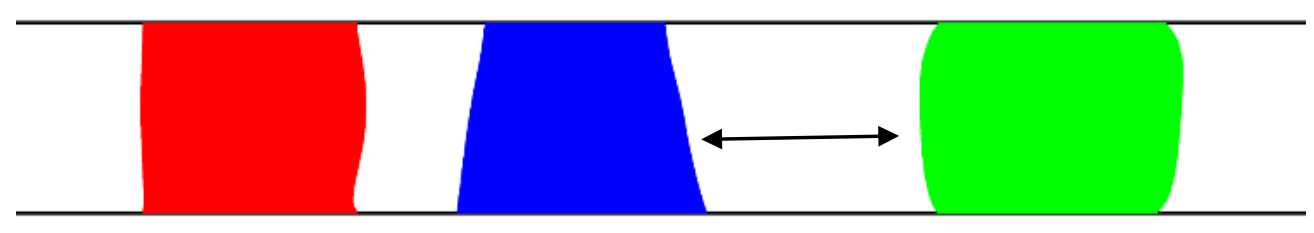

$112 \%$ spacing

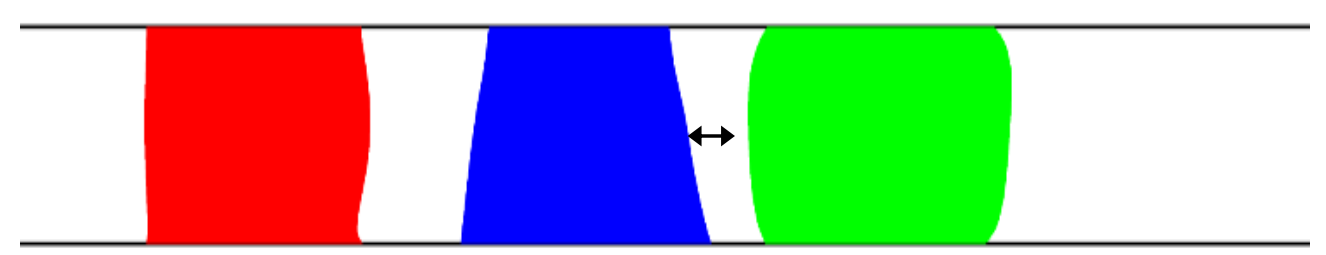

$29 \%$ spacing 


\section{Calculated effects of axial spacing between rotor and stator on total pressure rise}

1. Effects of non-uniform pressure field of stator on rotor performance.

2. Effects of rotor wake on stator performance ( wake recovery and wake/stator interaction). 


\section{Absolute pressure rise from rotor $\mathrm{LE}$ to stator TE}

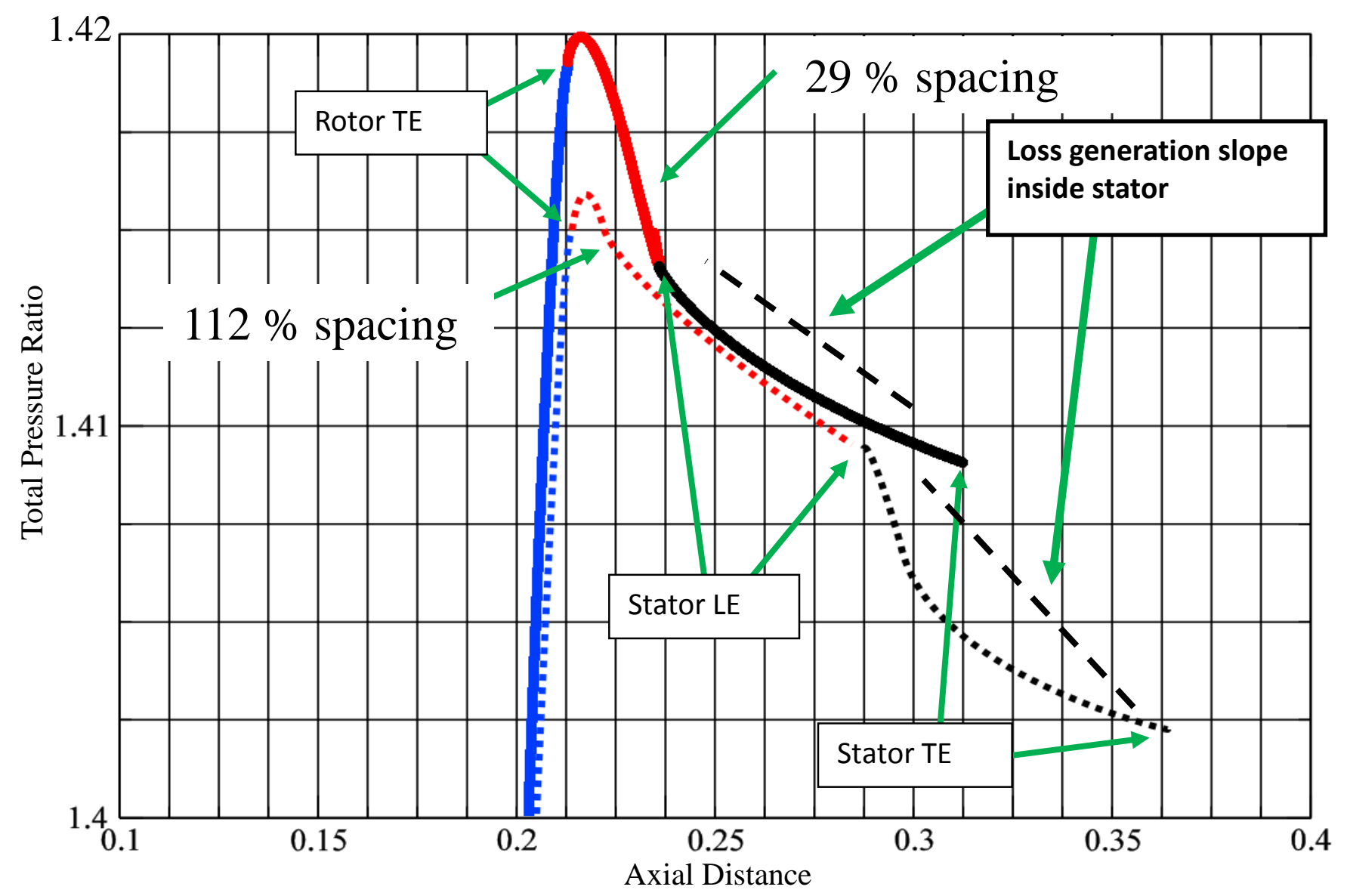




\section{Upstream effects of stator pressure field}

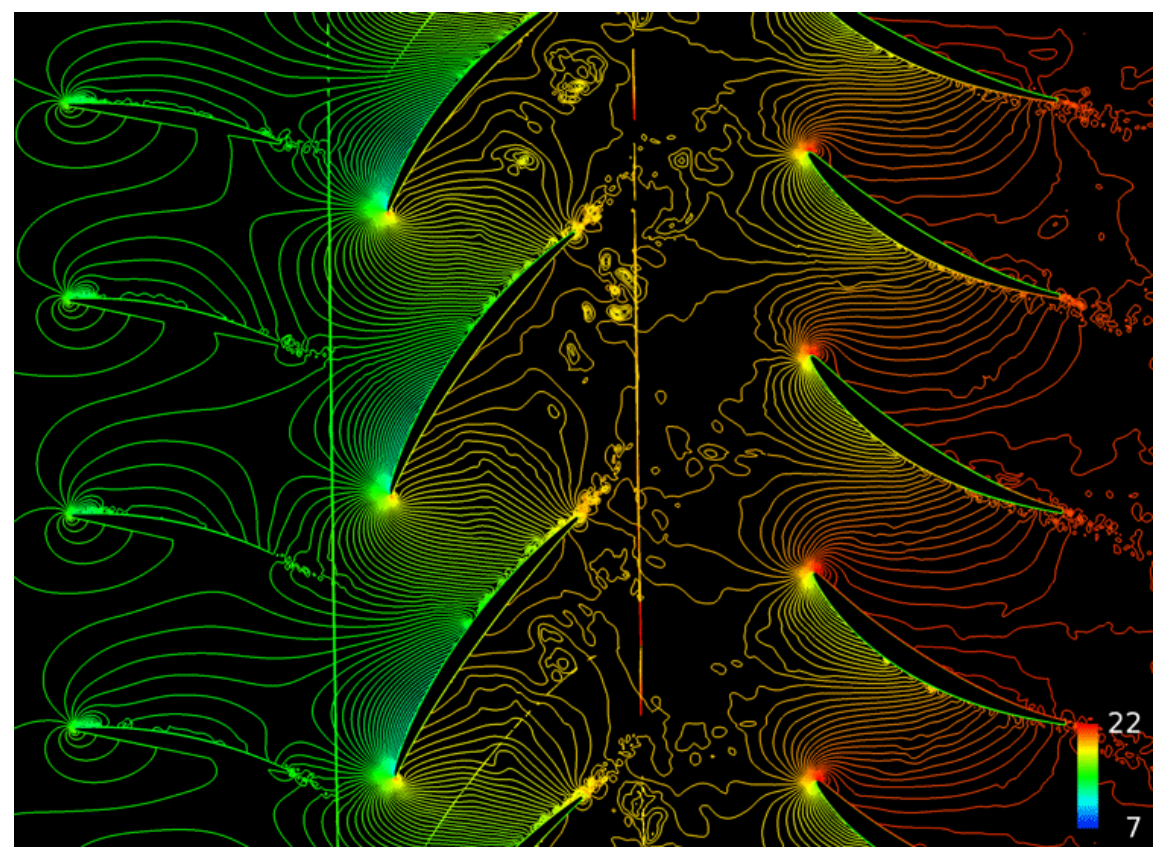

$112 \%$ spacing

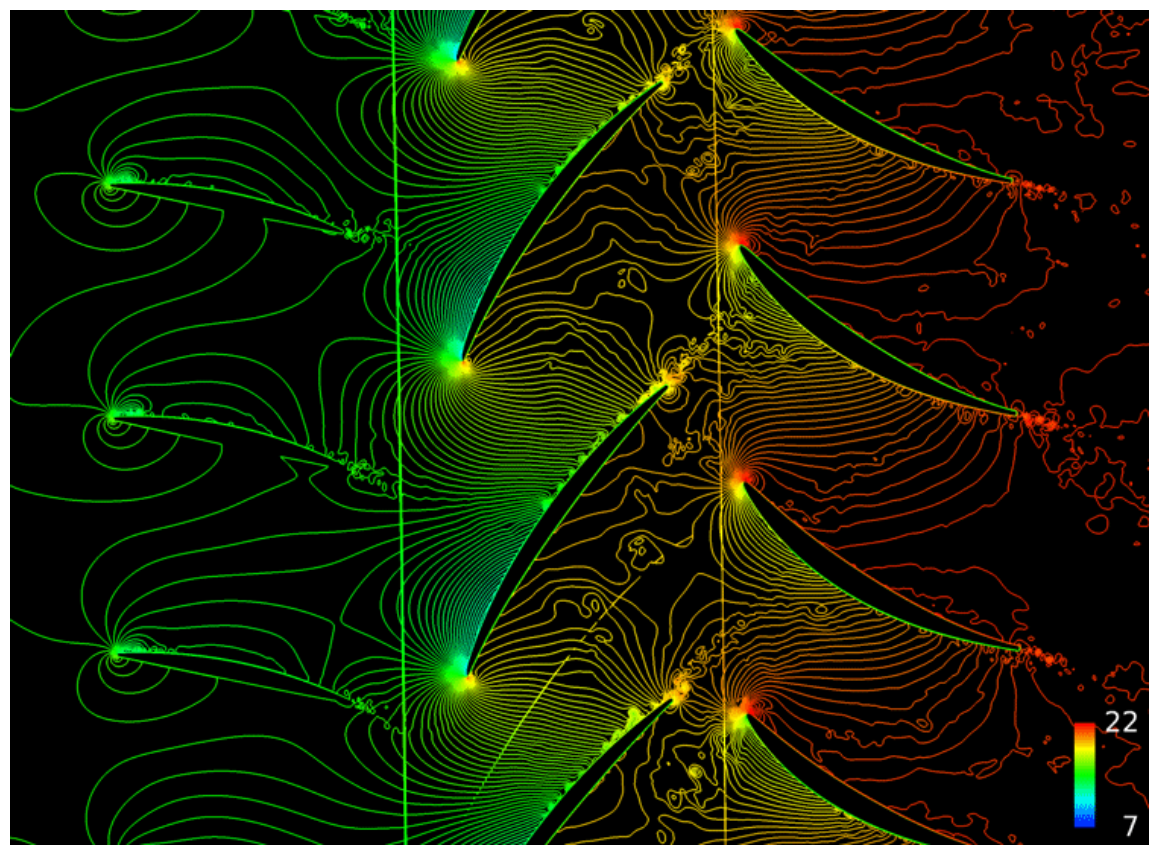

$29 \%$ spacing 


\section{Pt distribution due to wake dispersion}

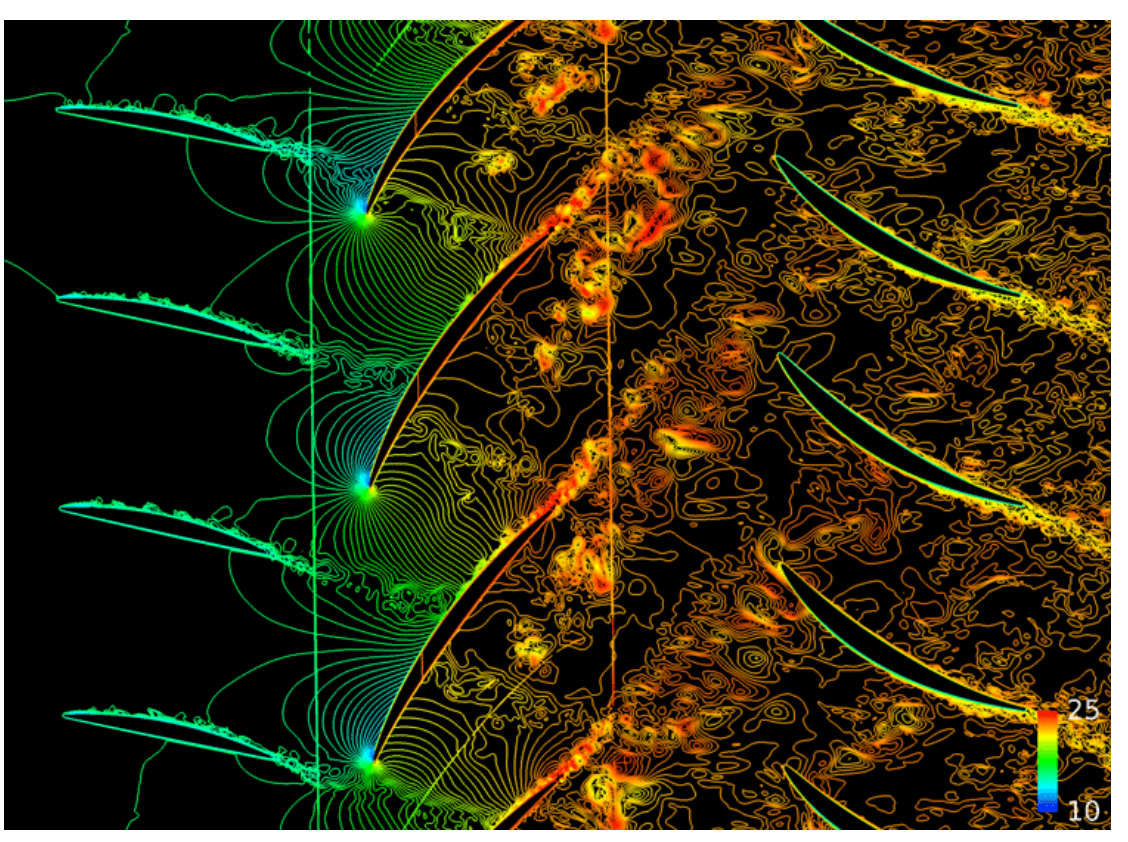

$112 \%$ spacing

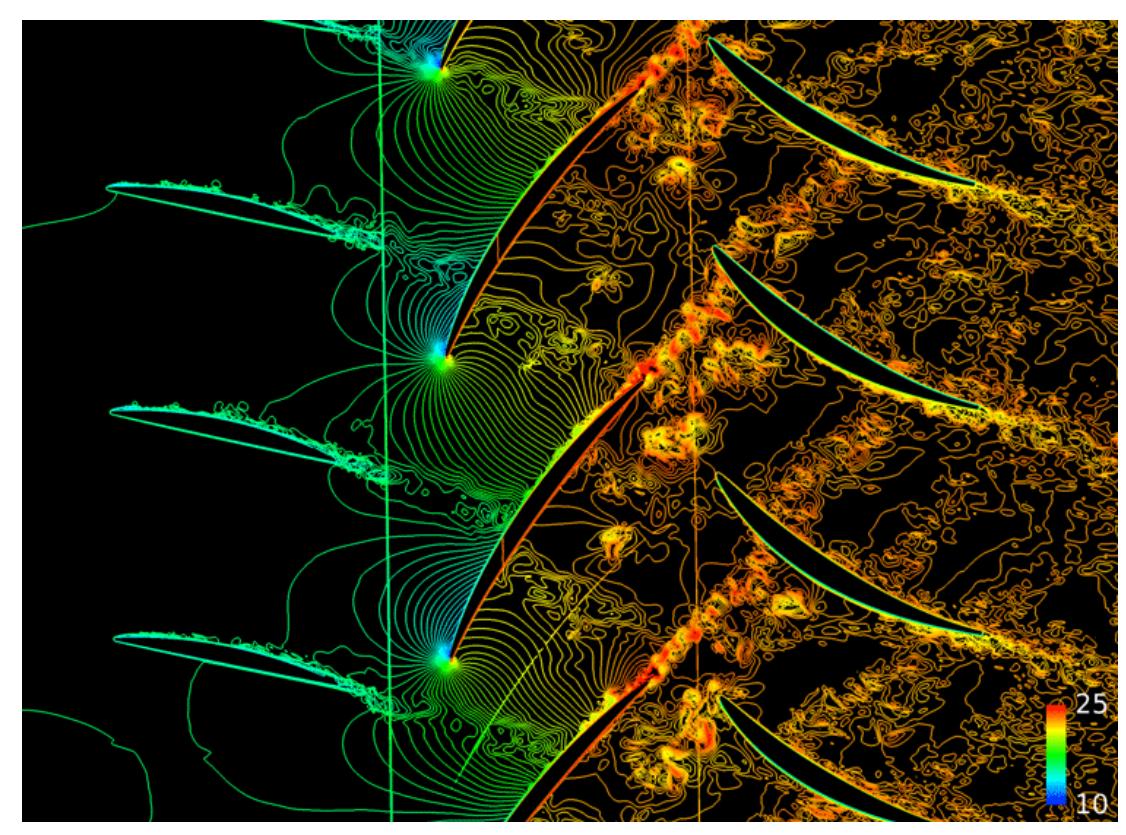

$29 \%$ spacing 


\section{Pt distribution at rotor exit}

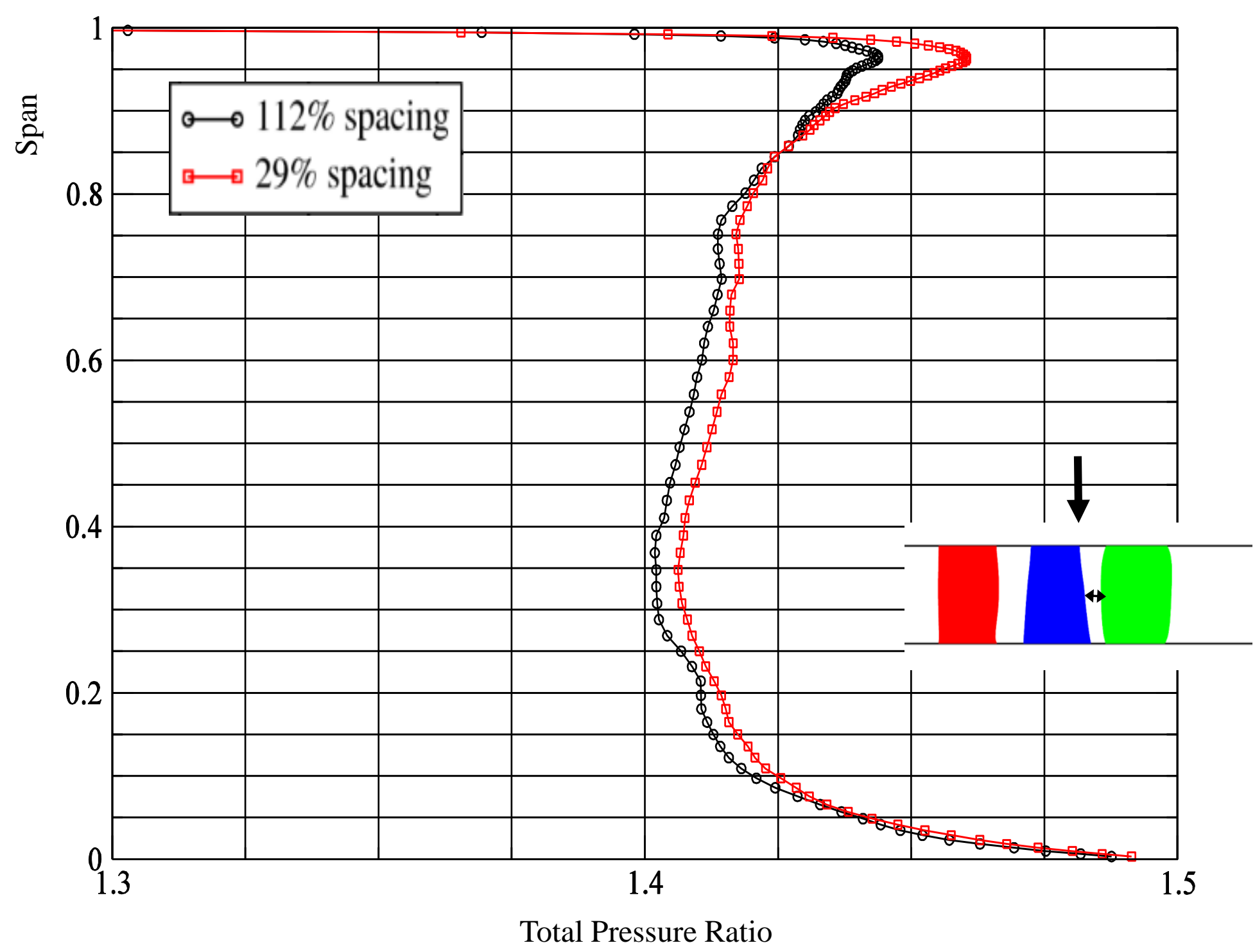




\section{Pt distribution at stator LE}

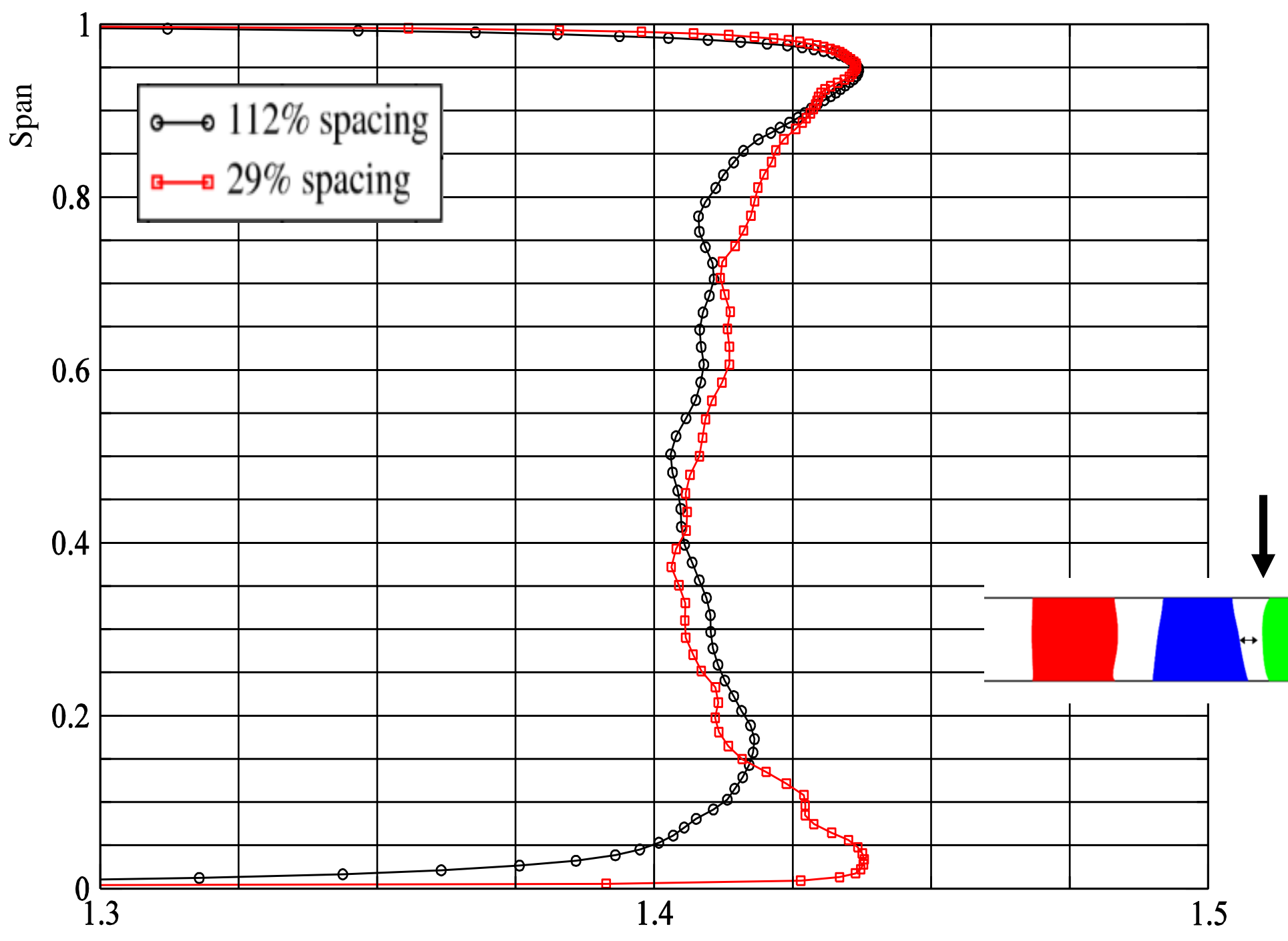

Total Pressure Ratio 


\section{Pt distribution at stator TE}

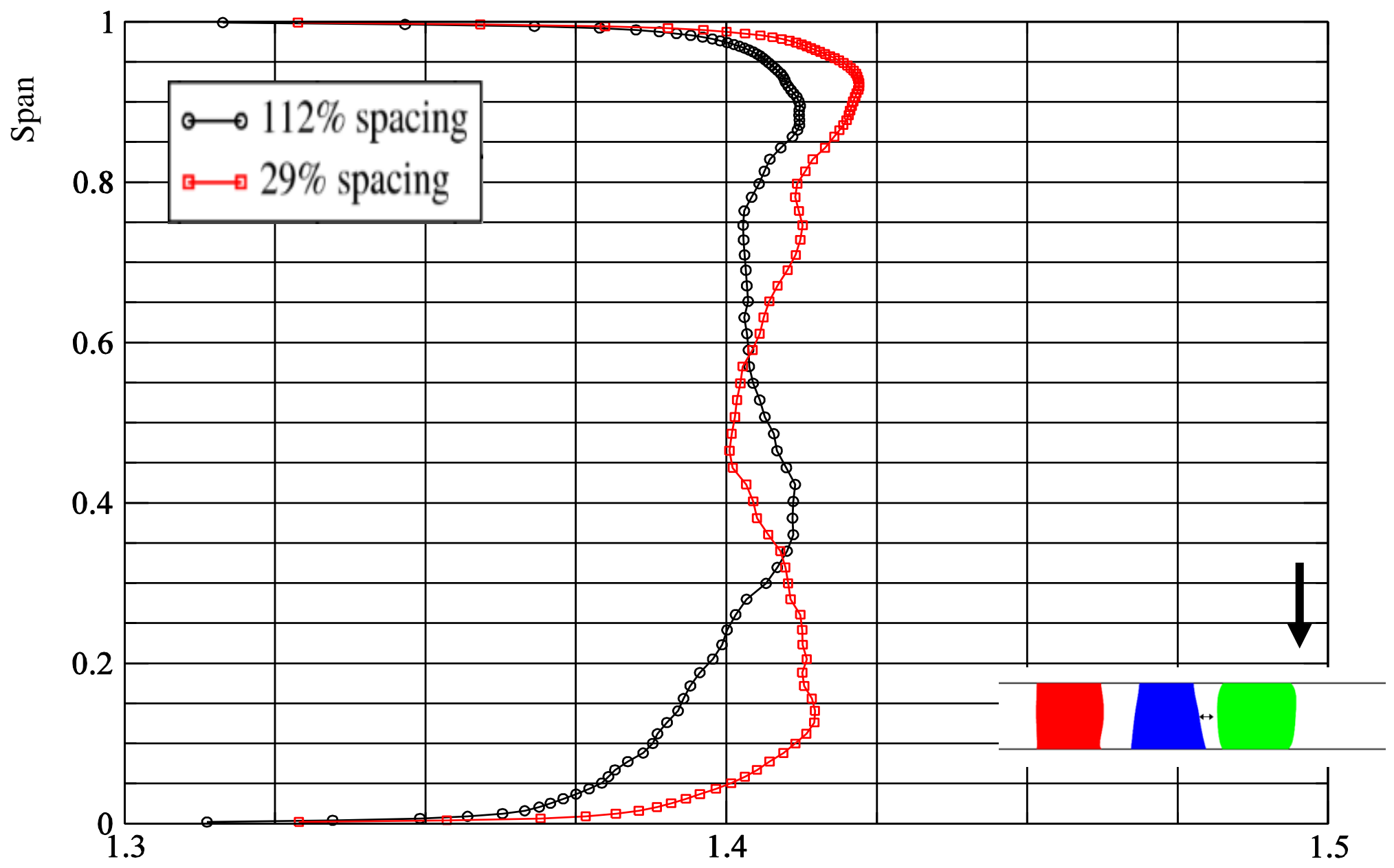

Total Pressure Ratio 


\section{Pt loss from Stator LE to TE}

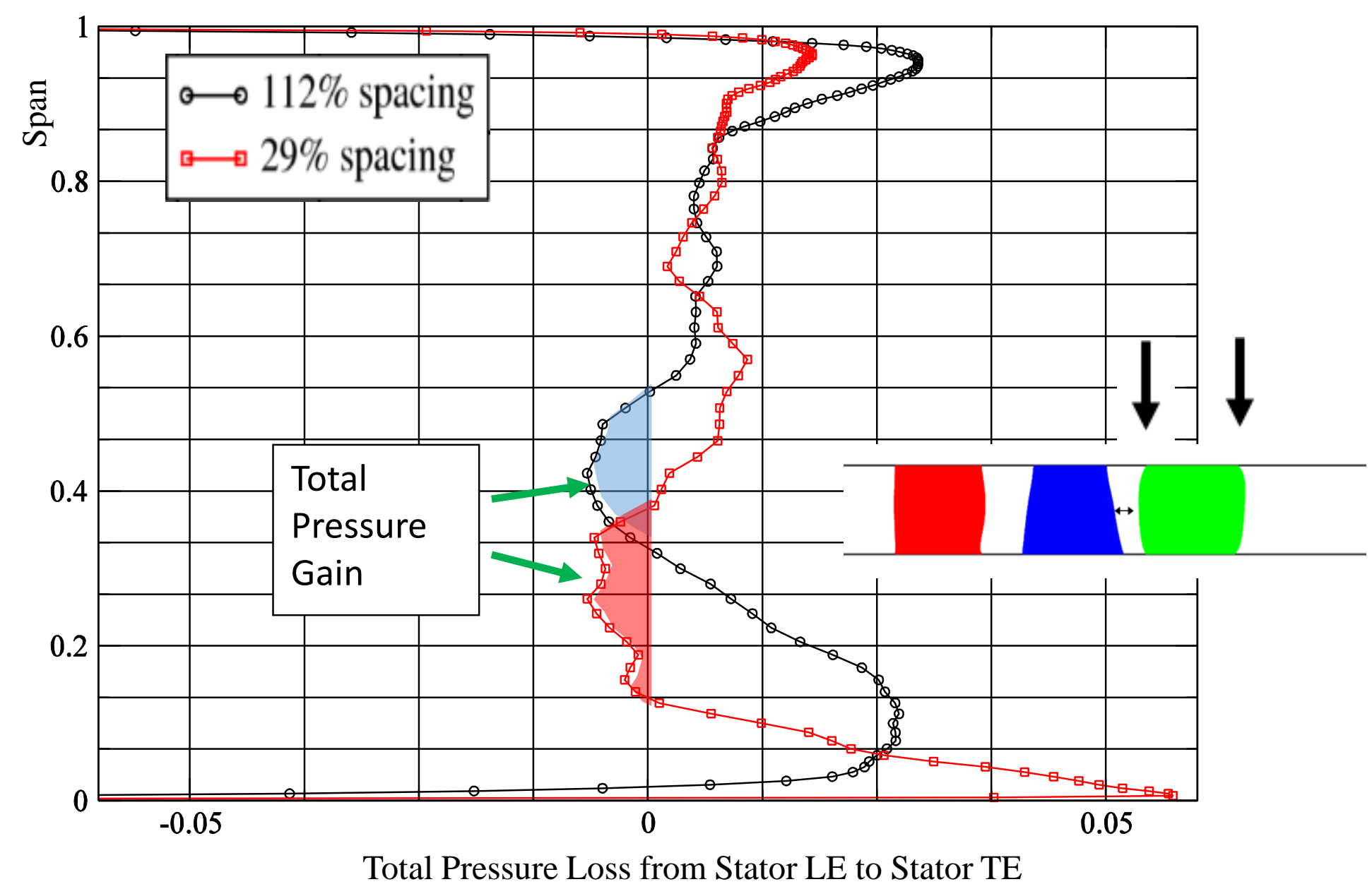


Breakdown of wake mixing loss from rotor TE to stator TE

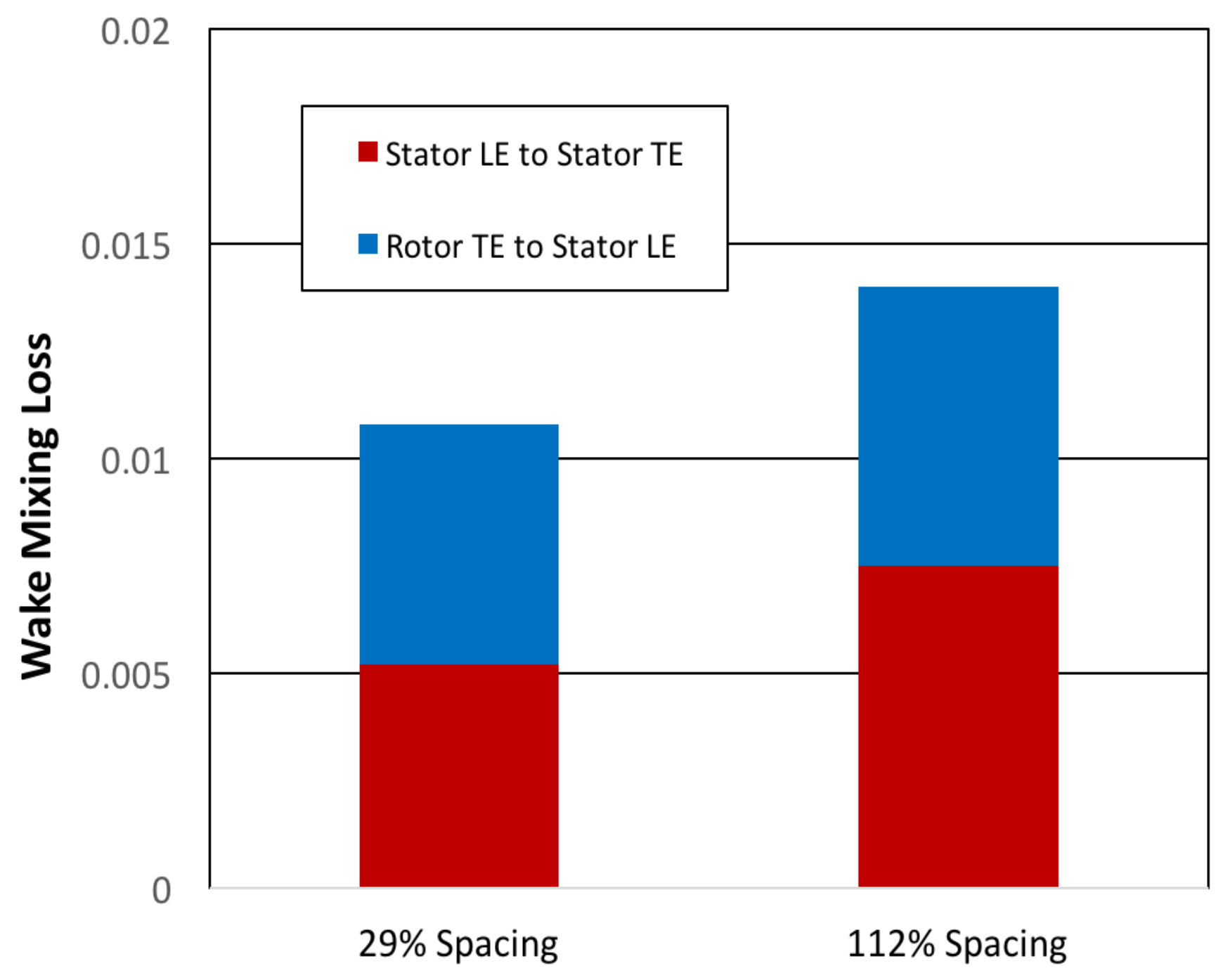




\section{Time-averaged $\mathrm{Pt}$ at stator TE}

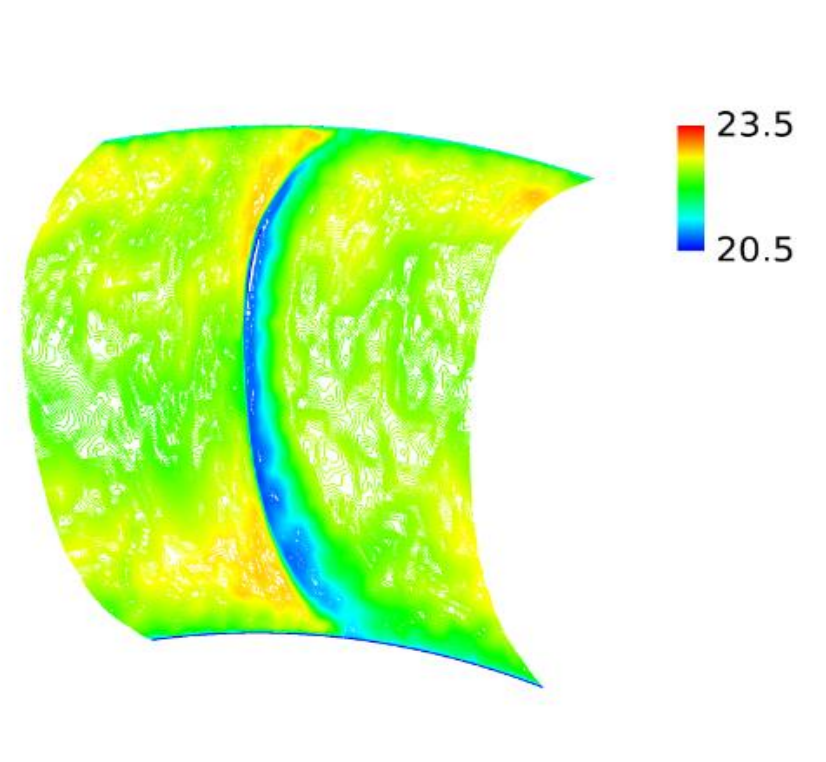

$29 \%$ spacing

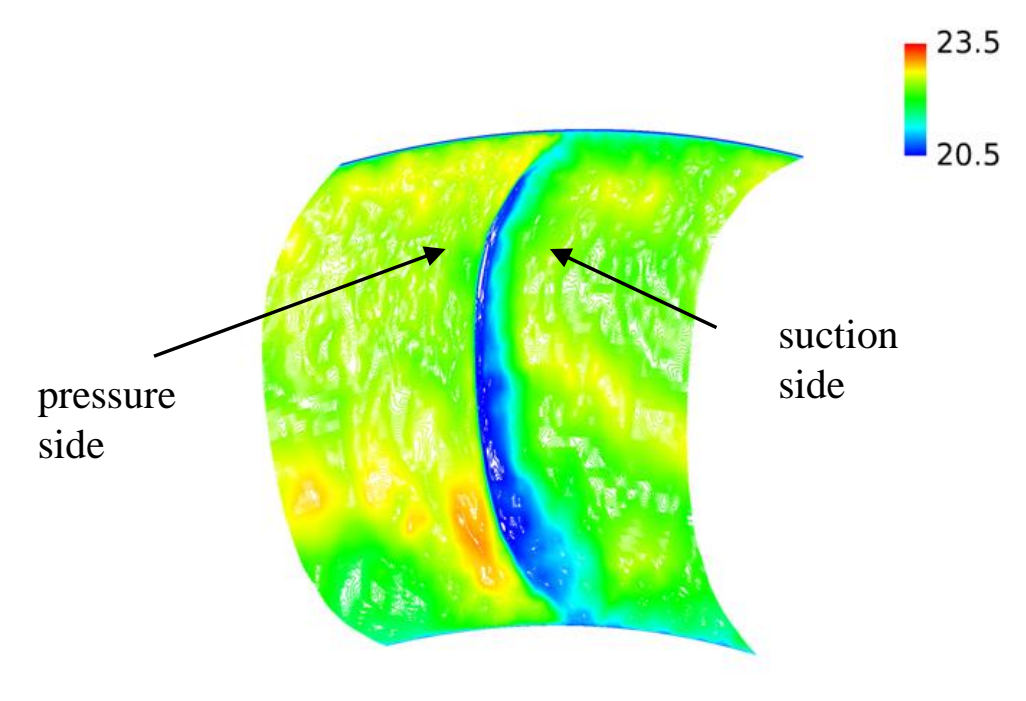

$112 \%$ spacing 
Measured Pt at stator exit, 1\&1/2 stage high speed compressor (Lurie and Breeze-Stringfellow[2015]

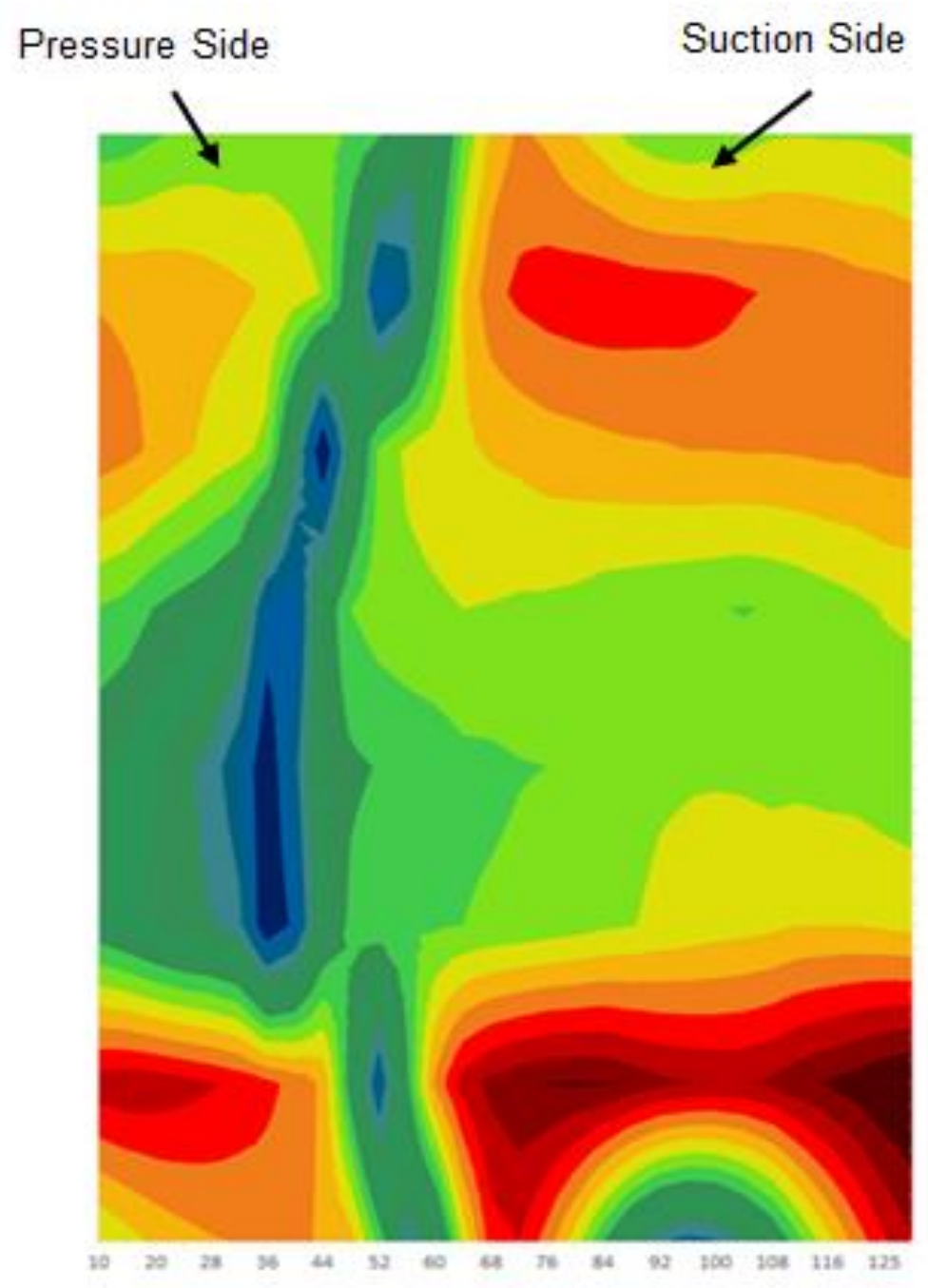




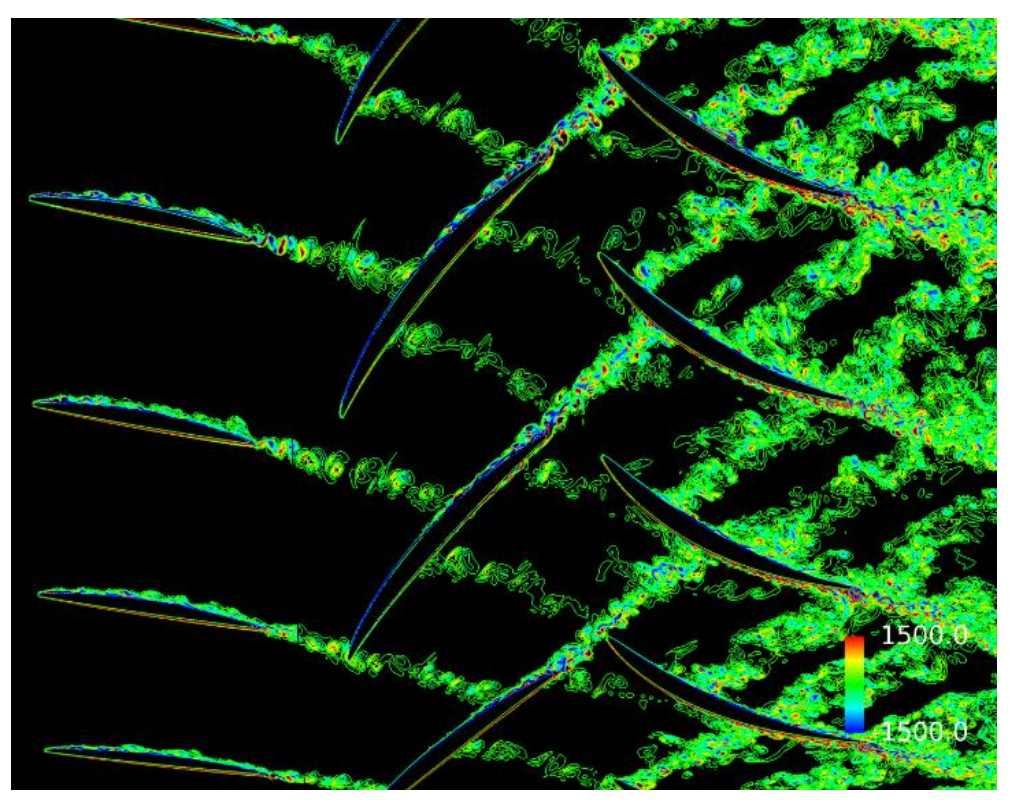

$30 \%$ Span
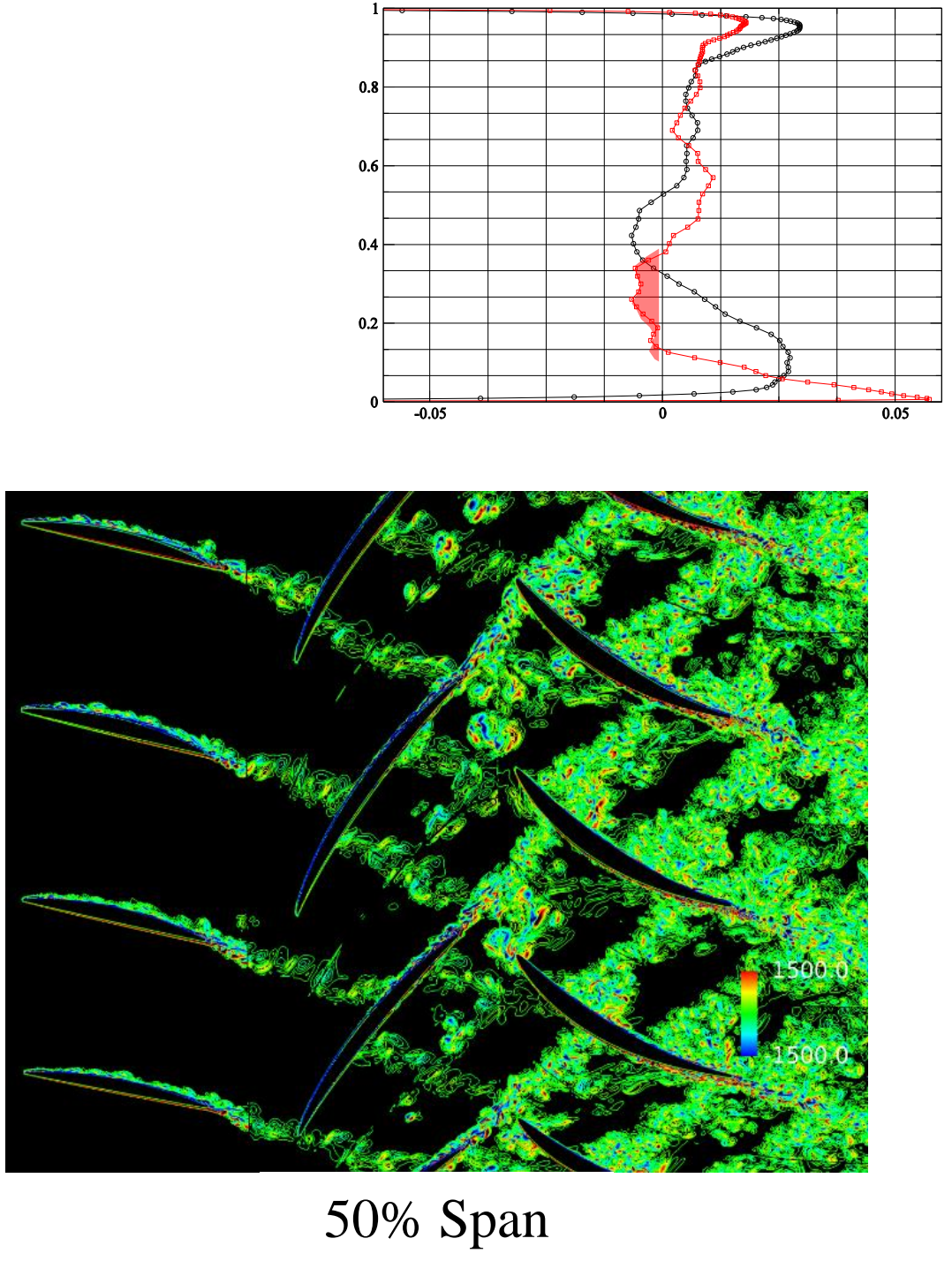


\section{Flow physics of wake recovery}




\section{Local strain rate of rotor wake inside stator passage}

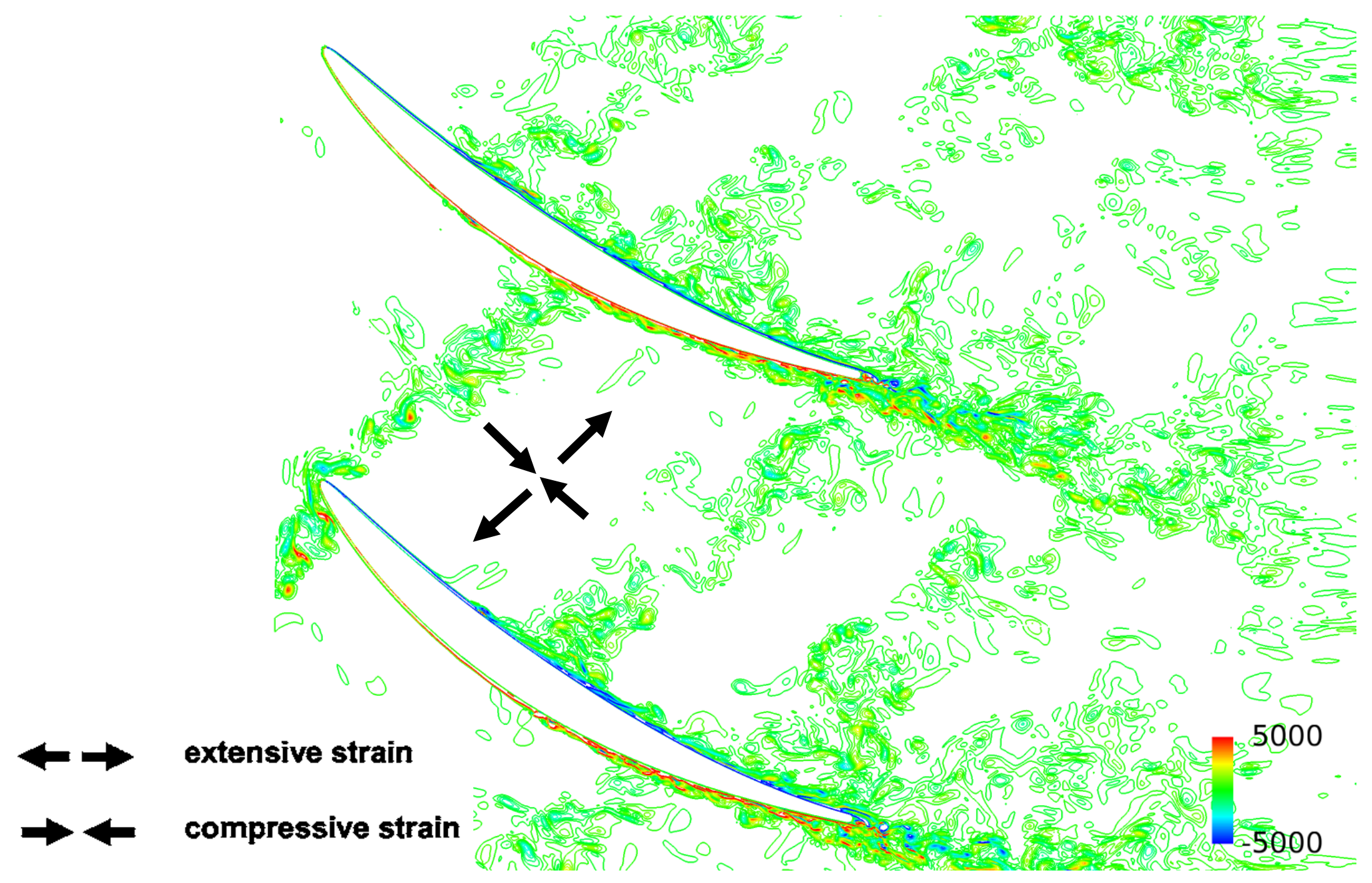




\section{Turbulence energy transfer through wake stretching}

1. Turbulence energy production. ( Soranna et al.[2006])

$$
P_{i j}=-\overline{u_{i}^{\prime} u_{k}^{\prime}} \frac{\partial \overline{U_{j}}}{\partial x_{k}}-\overline{u_{j}^{\prime} u_{k}^{\prime}} \frac{\partial \overline{U_{i}}}{\partial x_{k}}
$$

2. Wake stretching can transfer energy to different frequency domain.

3. Phenomena of small eddy interaction. 

$30 \%$ span, $29 \%$ spacing

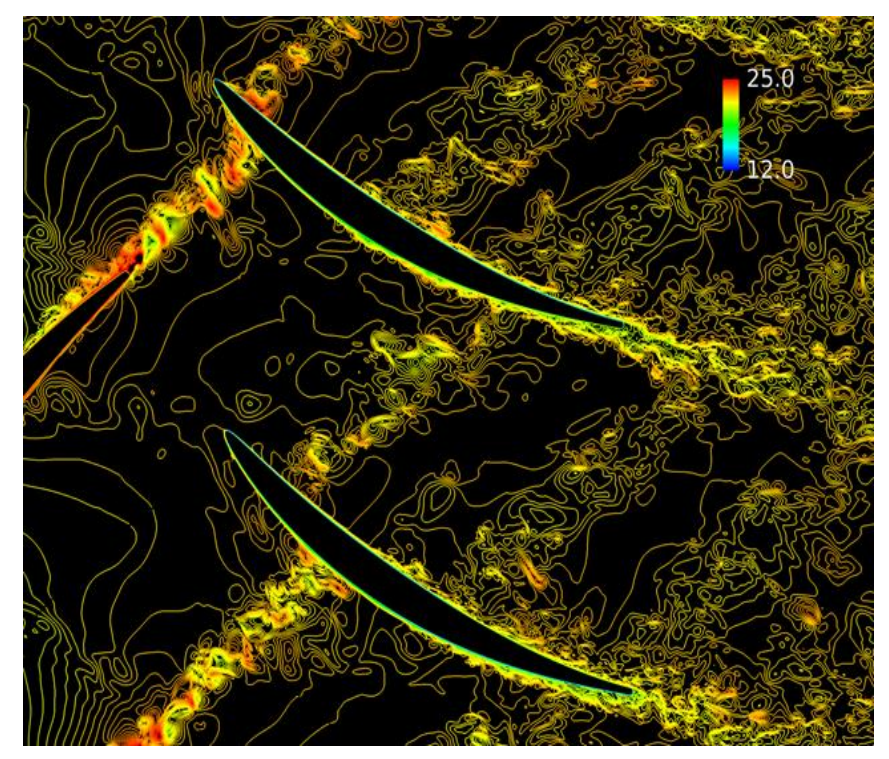

Original stator blade

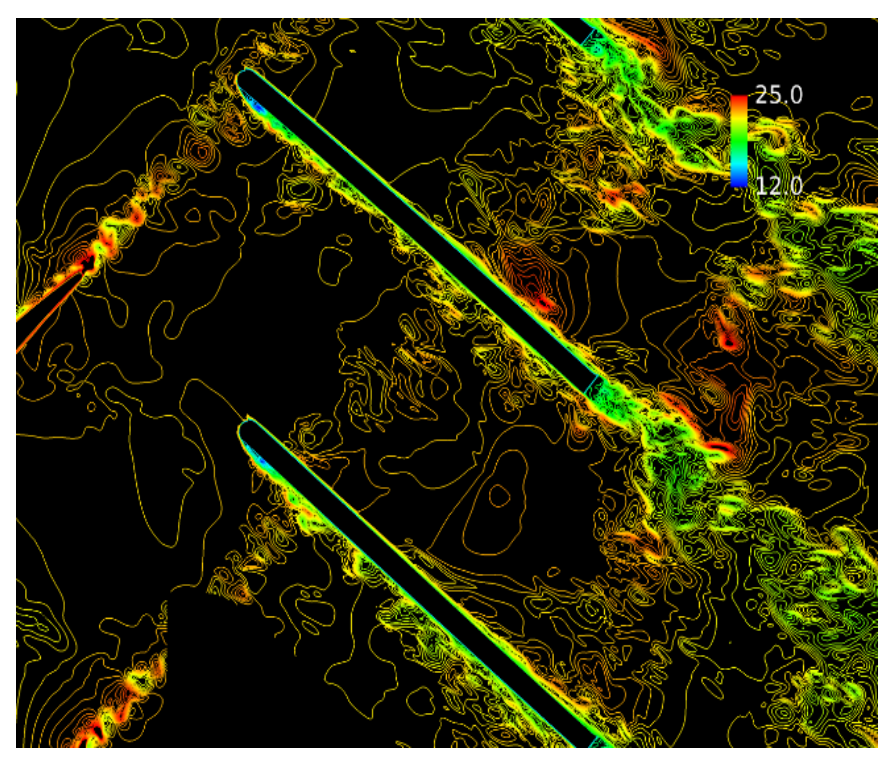

Flat plate blade 


\section{Concluding remarks}

- $0.5 \%$ Pt gain with the reduced spacing, 63 $\%$ is due to upstream pressure effect and 22 $\%$ is due to wake recovery.

- Wake recovery is due to energy transfer from turbulence to main flow. Turbulence production becomes negative due to opposing strains as wake stretches in nonequilibrium turbulence.

- Energy transfer occurs through small 3-D vorticities. 